\title{
REVIEWS
}

\section{Materials and technologies for soft implantable neuroprostheses}

\author{
Stéphanie P. Lacour1, Grégoire Courtine ${ }^{2}$ and Jochen Guck ${ }^{3}$
}

Abstract | Implantable neuroprostheses are engineered systems designed to restore or substitute function for individuals with neurological deficits or disabilities. These systems involve at least one uni- or bidirectional interface between a living neural tissue and a synthetic structure, through which information in the form of electrons, ions or photons flows. Despite a few notable exceptions, the clinical dissemination of implantable neuroprostheses remains limited, because many implants display inconsistent long-term stability and performance, and are ultimately rejected by the body. Intensive research is currently being conducted to untangle the complex interplay of failure mechanisms. In this Review, we emphasize the importance of minimizing the physical and mechanical mismatch between neural tissues and implantable interfaces. We explore possible materials solutions to design and manufacture neurointegrated prostheses, and outline their immense therapeutic potential.

'Bertarelli Foundation Chair in Neuroprosthetic Technology, Laboratory for Soft Bioelectronic Interfaces, Center for Neuroprosthetics, School of Engineering, Ecole Polytechnique Fédérale de Lausanne (EPFL), Switzerland. ${ }^{2}$ International Paraplegic Foundation Chair in Spinal Cord Repair, Center for Neuroprosthetics and Brain Mind Institute, School of Life Sciences, Ecole Polytechnique Fédérale de Lausanne (EPFL), Switzerland.

${ }^{3}$ Biotechnology Center, Technische Universität Dresden, 01307 Dresden, Germany.

Correspondence to S.P.L stephanie.lacour@epfl.ch

Article number: 16063 doi:10.1038/natrevmats.2016.63 Published online 27 Sep 2016
Ageing, trauma and neurodegenerative diseases lead to cognitive, behavioural and sensorimotor deficits that affect the quality of life of millions of people worldwide. Implantable neuroprostheses provide solutions to alleviate many of the symptoms associated with these conditions. For example, cochlear implants restore hearing in people suffering from profound hearing loss; deep brain stimulation alleviates Parkinsonian symptoms; spinal cord stimulators help manage neuropathic pain; and vagal nerve neuromodulation mitigates depression. Considering the number of patients who could benefit from these technologies, their dissemination remains limited and their potential remains comparatively underexploited. For example, paralysed individuals have been able to control a sophisticated robotic arm or their own forearm muscles using brain signals only ${ }^{1,2}$. However, the recorded neural signals extinguish rapidly ${ }^{3}$, which currently restricts the clinical potential of these implantable brain-machine interfaces ${ }^{4}$.

Historically, the biocompatibility of a neural implant has been mostly quantified in terms of chemical and electrochemical stability, and toxicity. However, tissueimplant interactions are triggered by very different phenomena, such as insertion trauma, neuroglial activation, cell migration and blood-brain barrier breakdown, that occur in concert and at multiple time points. In this Review, we explore one of these critical interactions that impedes the deployment of existing and contemplated implantable neuroprostheses: the physical and mechanical mismatch between man-made implants and the targeted neural tissues. Cells and neural tissues are several orders of magnitude softer than the materials used in neural implants. Cells respond to the mechanical properties of the surfaces they are in contact with. In vitro, environments with adjustable stiffness show that cells alter their motility, shape, differentiation outcome, activation and general function on the basis of the compliance and topography of their surroundings. In vivo, the soft neural tissues display viscoelastic properties that are often anisotropic, withstand uninterrupted motion due to blood flow, respiratory pressure and natural body movements, and undergo changes in volume throughout life. By contrast, most neural implants are rigid and static. For example, clinical implants are often prepared with platinum-iridium electrodes and stainless-steel wires embedded in silicone to form flexible, millimetrethick electrode paddles ${ }^{5-7}$. They may be machined with a built-in curvature to provide consistent electrode contact with the neural tissue. Such design is suitable for neurostimulation devices that require large electrode contacts over the surface of the brain or spinal cord. However, this type of technology fails to provide the resolution and stability that is necessary for long-term recordings of neural signals owing to the substantial distance between the electrode and targeted neurons. Implantable electrodes prepared in both academic environments and commercial research and development are prepared from a broader palette of materials borrowed from microelectronics, and biomedical and regenerative technologies. In the past decade, advances in soft bioelectronics - mechanically compliant electronics customized to interface biological tissues - have 
enabled novel strategies to remedy the issues of physical and mechanical mismatch and to restart the design process of implantable neuroprostheses ${ }^{8-10}$.

Here, we review recent strategies to design, manufacture and implement soft implantable neuroprostheses with improved biointegration and enhanced functionalities. We describe the shape, surface topology and biomechanics of neural tissues across mammalian species, and translate these physiological properties into design guidelines to construct physically matched synthetic implants. We provide examples of recent implantable neuroprotheses that have achieved superior neurointegration through designs, materials or technologies that obeyed at least one aspect of these guidelines. We conclude in discussing the enticing potential of soft implantable neuroprostheses to replace and restore neural functions after trauma and neurological disorders.

\section{Understanding neural materials Macroscopic and morphological considerations}

The mammalian central nervous system (CNS) is arguably the most complex biological organ. The CNS is composed of the brain and spinal cord, which send and receive signals to and from the rest of the body via the peripheral nervous system (PNS). Despite its peripheral location, the retina is also part of the CNS. The size and topography of the brain display remarkable differences across mammalian species (FIG. 1a). The adult human brain weighs approximately $1.5 \mathrm{~kg}$ (REF. 11) and the surface of the cerebral cortex is greater than $2,500 \mathrm{~cm}^{2}$. The brains of the main animal models used in neuroprosthetic research pale in comparison. For example, the weight of the brain and surface area of the cerebral cortex range from $1.8 \mathrm{~g}$ and $6 \mathrm{~cm}^{2}$ for the adult rat ${ }^{12}$ to $90 \mathrm{~g}$ and $110 \mathrm{~cm}^{2}$ for the rhesus monkey ${ }^{13}$. The topologies of the brain also differ widely: small mammals have smooth brain surfaces, whereas the brains of primates (including humans) display complex external topographies and patterns of convolution that result from gyrification of the cortical surface $^{14}$ (FIG. 1a). Gyrification refers to the cortical folding that gives the brain its wrinkle-like appearance with ridges (gyri) and grooves (sulci) ${ }^{15}$. The cerebral cortex of humans - the outer, 2-3- mm-thick layer of grey matter over the brain hemispheres - is a highly convoluted structure, with more than half of its actual surface area hidden from the apparent, directly accessible outer brain surface. Typical sulci radii and gyri depths in the cortex of primates ${ }^{13}$ are in the range of $1-5 \mathrm{~mm}$.

In the CNS, the cerebral and spinal tissues are protected by a multi-layered structure formed of meninges (that is, membranes including the pia mater, arachnoid mater and dura mater), bone, connective tissue and skin (FIG. 1b,c). In the PNS, longitudinal axon bundles are grouped in fascicles protected by the perineurium, which in turn may be bundled together with blood supply and fatty tissue within another sheath, the epineurium (FIG. 1d). Each of these membranes has a key mechanical role in protecting the delicate neural tissues and mitigating their dynamic response, not only to physiological movement and pressure changes in the cerebrospinal fluid, but also to injury.
Neural tissues withstand constant mechanical activity. The brain is a pulsatile organ submerged in cerebrospinal fluid and regulated by cardiac cycles and respiration. Adequate blood flow to the brain is crucial for maintaining normal brain function. Because the brain is enclosed in the rigid skull, pressure and flow pulsations are transferred to the soft brain tissue (resulting in local motion on a micrometre scale), arterial and venous network, and cerebrospinal fluid ${ }^{16}$. In freely behaving mammals, ventroflexion (that is, bending forward) causes tensile elongation of the spinal cord tissue accompanied with Poisson compression, whereas extension (that is, bending backwards) causes wrinkling of soft tissues inside the spinal canal. In humans, both the spinal cord and its meningeal protective membranes can experience as much as $10-20 \%$ tensile strain and displacement (relative to the spinal canal) during normal postural movements. This motion corresponds to displacements on the order of centimetres ${ }^{17}$. The deformations relative to the spinal cord in animal models, such as rodents or non-human primates, are likely to be even larger.

The mechanical properties of the dura mater and the nerve epineurium and perineurium are especially relevant for neural implants, because most interfaces lie immediately above or below this protective membrane or must pierce through it in order to access the neural tissue. The dura mater consists of collagen fibres that are interdispersed with fibroblasts and elastin and is traversed by a network of blood vessels. In humans, its thickness varies from 0.3 to $0.8 \mathrm{~mm}$, depending on the age of the individual ${ }^{18,19}$. Both cranial and spinal dura maters exhibit hyperelastic and viscoelastic stress-strain behaviour, typical of collagenous tissues, with an elastic modulus of 0.5-1.2 MPa (REF. 20). Their toughness also prevents the insertion of brittle electrodes through the dura mater without performing a surgical incision of the meninges and/or the use of guide tubes or cannulas.

Peripheral nerves are anisotropic structures that spread throughout the body. They vary significantly in diameter; for example, the small visceral nerves are about 100 times smaller than the sciatic nerve, which reaches up to $2 \mathrm{~cm}$ in diameter in humans. Nerve epineurium and perineurium are formed of bundles of collagen fibres and elastin ${ }^{21}$. When tension is applied to a nerve, its initial undulated resting shape accommodates some elongation without generating appreciable stress along the fascicles and axons. The elasticity of the connective tissue surrounding a nerve helps preserve the integrity of the axon. As the tensile loading increases, the nerve displays quasi-elastic stress-strain behaviour until rupture of the perineurial sheath, which occurs with a fracture strain of $\sim 25 \%$ and an ultimate stress of $\sim 10 \mathrm{MPa}$. Nerve fibres start to rupture in the endoneurium sheath at lower strains ${ }^{21}$.

\section{Cellular considerations}

The brain and spinal cord host different kinds of neurons and several types of glial cells, which interact in many intricate and mutually supporting ways to enable the overall functional performance of the neural tissue. 
a Relative brain size and complexity
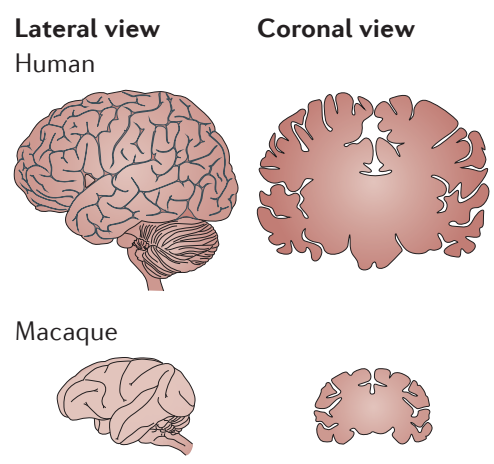

Rat

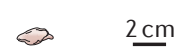

a c Spinal cord

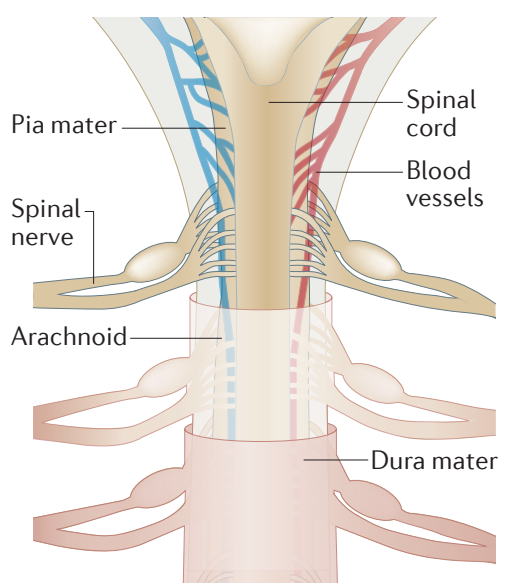

b Protective layers around the brain

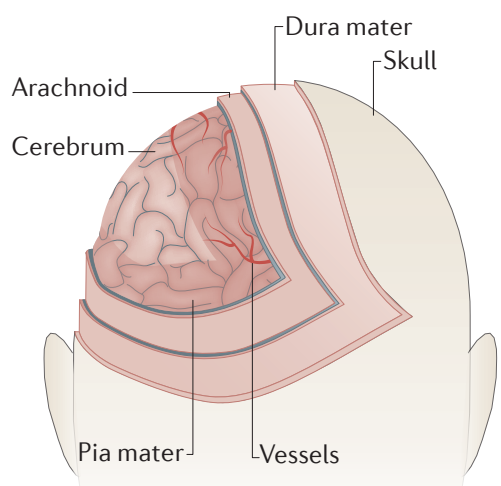

d Peripheral nerve

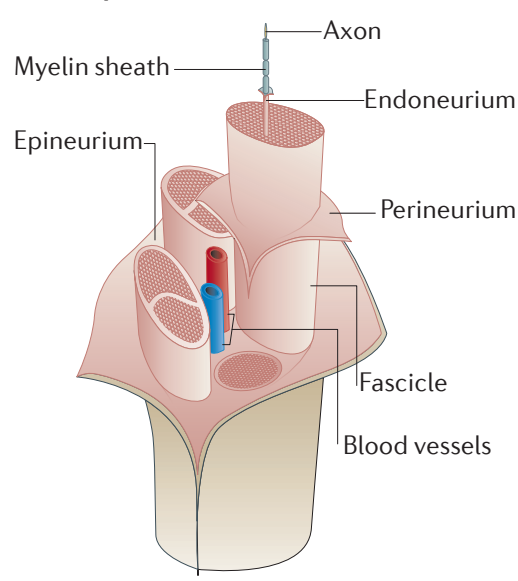

Figure 1 | Structure and anatomy of the nervous system. a| Schematic illustrations of the lateral and coronal views of the brain across mammalian species. Illustrations of the anatomy of the human brain (part b), the spinal cord (part c) and a peripheral nerve (part d) are also shown. body reaction (FBR) against foreign material, such as implants, introduced into the $\mathrm{CNS}^{22}$.

The material properties of these cellular tissue constituents contribute to the stiffness of the entire tissue. Their intrinsic properties have been investigated ${ }^{23,24}$ after isolation from the tissue. Neurons, isolated from neonatal rat cortex, guinea-pig hippocampus and retina, have been probed with atomic force microscope (AFM)-based nano-indentation, which revealed rate-dependent, nonlinear viscoelastic behaviour with shear moduli between a few tens to a few hundred pascals ${ }^{25-27}$. In these studies, it was found that under lateral compression, neuronal and glial processes were softer than their somata ${ }^{26}$. However, a more recent study using magnetic tweezers for local rheological measurements revealed that the soma is softer and more solid-like compared with the stiff and viscous-like neurites. The authors concluded that axons are probably more sensitive to mechanical damage than the soma ${ }^{28}$. However, the main load-bearing direction of neural processes is actually oriented along their length, in which elastic spring constants of a few hundred $\mathrm{mN} \mathrm{m}^{-1}$, viscoelastic relaxation and also active contraction have been observed ${ }^{29-34}$. It is noteworthy that processes of neurons in the CNS display lower spring constants than those of neurons in the $\mathrm{PNS}^{8,22-24}$. In contrast to many other cell types, glial cells are roughly as compliant as neurons with Young's moduli on the order of ten to a few hundred pascals ${ }^{26,35,36}$. Relevant in the context of the FBR against implants is that cell stiffness can change when cells are activated. For example, acutely isolated Müller glial cells from an ischemic retina are stiffer than the cells from a healthy retina. This increased stiffness is attributable to an increase in the density of intermediate filaments such as glial fibrillary acidic protein (GFAP) and vimentin ${ }^{36}$. By contrast, cultured primary astrocytes soften considerably following either interferon- $\beta$ (IFN $\beta$ ) stimulation ${ }^{37}$ or a stretch-induced upregulation of GFAP ${ }^{38}$. To our knowledge, no information on microglial stiffness has been reported so far, which is probably because of the phagocytic activity of these cells when they are in contact with a measurement probe. and between 1 and about 1,000 dendrites, which receive signals via synapses from other nerve cells. After integration and processing of this external information, action potentials propagate towards other cells via a single, long process called the axon. The axon is a few micrometres thick, but can reach up to a metre in length. The network of neuronal processes conveys a considerable amount of mechanical connectivity throughout the tissue. The function of the various glial cell types is to support and modulate the performance of the neurons. Oligodendrocytes (similar to Schwann cells in the PNS) produce thin and wide insulating myelin sheaths wrapped around each axon. These cells primarily support the transport of information along the axon, but also offer additional mechanical connectivity to the tissue. Astrocytes provide general biochemical support to other cells and modulate the information processing at synapses. They also contribute to the repair of tissue after injury and the formation of scars that protect the neural environment from the rest of the body. Microglia are the resident immune cells of the CNS, which also initiate and mediate the foreign

\section{Tissue considerations}

The extracellular matrix (ECM) is embedded in the interstitial space between the cell bodies and their processes. The ECM consists of fibrous proteins, proteoglycans and glycosaminoglycans, such as laminin and hyaluronan. In the CNS, these elements form a dense mesh ${ }^{39-42}$ with a striking scarcity of collagen, which is the dominant ECM CNS, the total volume fraction of the ECM is about $20 \%{ }^{44}$. For a recent review of the regional distribution of these ECM components and the corresponding cell adhesion molecules in the CNS, see REF. 45. In addition to their direct biological function, the ECM molecules maintain adhesion between cells and thus provide additional mechanical resistance to the deformation of the entire tissue.

How these components - cells and the ECM contribute to the overall mechanical properties of the CNS tissue is still poorly understood. Their relative importance is probably inverted compared to that of component elsewhere in the body ${ }^{43}$. In the adult human 
most other soft tissue in the body, for which the stiffness is largely dominated by a polymer matrix formed of collagen with the interstitial cells having less importance. Consequently, both collagen gels reconstituted in vitro and most body tissues display strain stiffening and compressive softening. Brain (and fat) tissue exhibits the opposite mechanical behaviour: it stiffens under compression but softens with strain ${ }^{46}$. This behaviour is consistent with the picture of the brain as largely a dense colloidal system (in which the cell bodies are the colloids) connected by an adhesive mesh of ECM, direct cell-cell contacts and connections via cell processes (for example, dendrite, axon and glial processes). The overall CNS stiffness is comparable to that of individual cells and, for example, in the brainstem, axons have been shown to be stiffer than the surrounding $\mathrm{ECM}^{47}$. Further, as previously mentioned, collagen is absent from the ECM of the brain, which only takes about $20 \%$ of the volume and thus is unlikely to have a large load-bearing role. Both neural cells and ECM are nonlinear mechanical entities that can alter their properties under isotropic pressure and anisotropic tension or compression ${ }^{48}$. In addition, $\mathrm{pH}$ and ions in the interstitial liquid, together with their interplay with the highly charged and sulfated ECM components, contribute osmotic and additional charge effects ${ }^{49}$.

Despite the current lack of a comprehensive, bottomup model of the mechanical properties of CNS tissue, there is no scarcity of experimental reports on the mechanical properties of brain, spinal cord and retina, both in vivo and in vitro, and at spatial scales from organ to cell. Since the first measurements in the $1960 \mathrm{~s}^{50,51}$, there are now several excellent reviews covering the wide spectrum of work conducted on this topic $\mathrm{c}^{23,48,49,52}$. For the bulk brain, typical elastic moduli reported range from a few $100 \mathrm{~Pa}$ to about $10 \mathrm{kPa}$ in oscillatory shear rheological measurements in vitro, depending on strain, shear rate, preconditioning and several other factors ${ }^{48}$. This also holds for the spinal cord ${ }^{48,53}$ and retina ${ }^{54}$.

On a smaller scale, depending on the region of the CNS, different types, spatial orientations and relative numbers of neurons, glial cells and their processes, as well as different amounts of ECM, contribute to the mechanical properties measured ${ }^{53,55-59}$. For example, grey matter regions in the cerebellum, consisting mainly of cell bodies, tend to be stiffer (Young's modulus measured with $\mathrm{AFM}, E \approx 450 \mathrm{~Pa}$ ) than white matter regions $(E \approx 300 \mathrm{~Pa})$, which essentially contain many axonal $\operatorname{tracts}^{60}$. A similar result has been reported for the rat spinal cord measured with AFM, further demonstrating the greater anisotropy of white matter ${ }^{53}$. Even on a larger scale, this result seems to hold ${ }^{61}$. The difference between white and grey matter might stem from the lower cell density in the former ${ }^{53}$, which is structurally dependent on the oligodendrocyte connectivity, because demyelination in the rat spinal cord leads to lower stiffness and tensile stress ${ }^{62}$. Lower stiffness has also been reported for demyelinated regions in the murine brain parenchyma measured with magnetic resonance elastography $(\mathrm{MRE})^{63}$. Although this seems to form a clear picture, it should be noted that there are also reports of white matter being $40 \%$ stiffer, and more viscous, than grey matter ${ }^{55}$.
Age can also have a role, with older brains tending to be stiffer $^{64}$. By contrast, MRE shows fluidization with age (and that female brains are more elastic than male brains $)^{65}$. Such discrepancies are not uncommon in this field and are widely discussed ${ }^{22,47,51,65,66}$. They could emanate from different preparative procedures of the tissue, time and length scales, as well as modes of deformation, and the change of properties between in vivo and in vitro measurements, among other possible factors. Improved microscopic models of brain tissue might help to resolve these differences in the future $e^{49,66}$.

\section{Important features for neural implant design}

Given the uncertainties discussed in the previous subsections, what are the reliable features and what do we need to consider in the design of neural implants? One important parameter is the compliance of the neural tissue, quantified by its Young's modulus and reported in the range of $100 \mathrm{~Pa}$ to $10 \mathrm{kPa}$ for CNS tissue. In contrast to other biological tissues, the high compliance of the CNS tissue is independent of species, age and regions within the CNS. Another important feature to consider when evaluating materials properties for the design of future implants is the size. Indeed, tissue stiffness values strongly depend on the probing technique, associated probe format and rate at which the tissue is strained. For example, the lowest values of about $100 \mathrm{~Pa}$ obtained by $\mathrm{AFM}^{53,57,60,64}$ for the CNS modulus are probably due to tissue constituents, and importantly water, being able to accommodate and flow around the micrometre-sized, indenting probe. On scales much larger than that of the tissue constituents, or in tensile probing, the incompressibility of water will probably contribute a much greater resistance to motion and thus yield a higher elastic modulus ${ }^{67}$. Consequently, it could be possible for large implants to be kept stiffer (on the order of $10 \mathrm{kPa}$ ) than implants with fine features on a cellular scale, such as thin meshes or micrometre-scale wires.

A third parameter to consider is the mechanical contrast between the pulsating neural tissue and the static implant. The recurrent motion of the surrounding tissue with respect to implants will cause different kinds of damage, depending on the physical properties of the implant ${ }^{68}$. The insertion of the implant into the brain also alters the mechanical properties of the surrounding tissue itself. After the implantation of microelectrodes, brain tissue exhibits appreciable stiffening during the first four weeks after implementation owing to the formation of a stiff matrix surrounding the implant. This matrix gradually softens and the stiffness eventually returns to its original value (that is, before implantation) ${ }^{68}$. A more extreme example is the effect of a lesion on neural tissue. For example, the hemisection of a rat spinal cord leads to a decrease of the elastic modulus but an increase of the viscosity, measured by micro-indentation 2 and 8 weeks after injury ${ }^{69}$.

The development of most neural implants is conducted in mammalian animal models. Therefore, the similarities and differences in the mechanics, morphology and size of relevant tissues between the most common animal models and humans must be taken into consideration. In a study ${ }^{70}$ published in 1970 , it was 


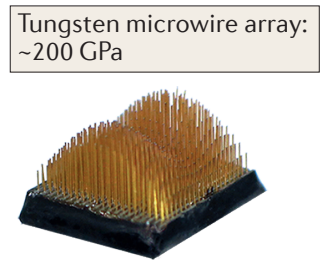

E

$\square$ Biological tissue

$\square$ Neural implants
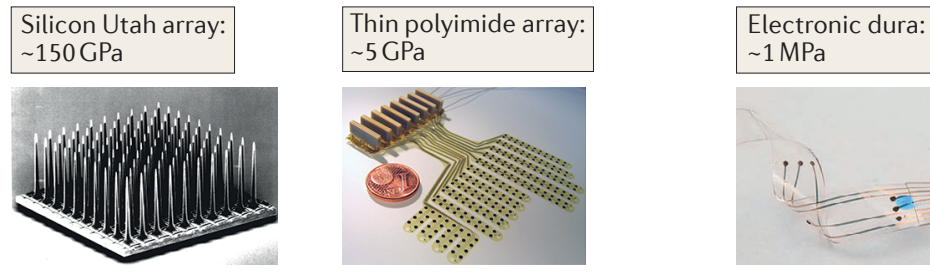

$\sim 1 \mathrm{MPa}$

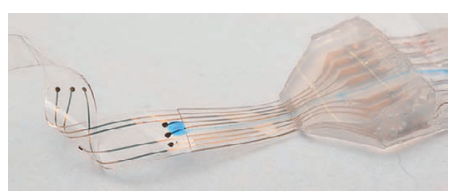

$100 \mathrm{GPa}$

$1 \mathrm{GPa}$
$1 \mathrm{kPa}$
Cortical bone: $10 \mathrm{GPa}$
Dura mater: $\sim 1 \mathrm{MPa}$
Spinal cord and brain: $100 \mathrm{~Pa}$ to $10 \mathrm{kPa}$

Figure 2 | Mechanical mismatch between the nervous tissues and man-made implantable electrodes. Soft neural tissues are wrapped in elastic dura mater and encased in hard bone sheath. Neural electrodes are traditionally made of stiff microelectronic materials (for example, metals such as tungsten microwires, inorganic semiconductors such as Silicon Utah arrays ${ }^{123}$ and plastics such as polyimide arrays ${ }^{124}$ ) and have only recently matched the dura mater mechanical characteristics ${ }^{20}$. E, Young's modulus. Image of the tungsten microwire array $\left(5 \times 5 \mathrm{~mm}^{2}\right)$ is courtesy of Tucker-Davis Technologies. Image of the silicon Utah array $\left(2 \times 2 \mathrm{~mm}^{2}\right)$ is reproduced with permission from REF. 123 , Elsevier. Image of the polyimide array is reproduced with permission from REF. 124, Institute of Physics. Image of the electronic dura (implant width: $5 \mathrm{~mm}$ ) is reproduced with permission from REF. 20, AAAS.

found that human tissue is slightly softer than that of the rhesus monkey. However, the limited and sporadic availability of CNS tissue has restricted the evaluation of tissue mechanics ex vivo in humans. Consequently, no systematic comparison between human and other species is available. If the mechanical properties can safely be assumed to be very similar, one aspect that does differ considerably is the relevant sizes involved. Whereas a mouse brain has a diameter of about $1 \mathrm{~cm}$, a human brain is about 15 times larger (FIG. 1a). Other CNS features and macroscopic curvatures exhibit similar differences in scale among species. These differences pose design considerations in terms of the bending stiffness (thickness) of implants when scaling up from small animal models to implants for use in humans. This is especially true when implant conformity to the increasingly undulated brain surfaces from mouse to primate brains is important.

We are still far away from a having a thorough understanding of the complex and intricate mechanical properties of CNS tissue, which itself is an interesting frontier of materials research. However, we probably know enough to start tailoring implants that match the mechanics of the brain. In the following section, we highlight recent technological efforts geared towards implementing mechanical compliance in synthetic materials and implants.

\section{The soft neurotechnology toolbox}

Today, there is no worldwide consensus on the most appropriate implantable materials for neural interfaces. Platinum-iridium, titanium, stainless steel and silicones are widely used in clinical devices following stringent and local standards regulated, for example, by the International Organization for Standardization (ISO) in Europe, the US Food and Drug Administration (FDA) and the Ministry of Health, Labour and Welfare (MHLW) in Japan. It is also important to consider that an approved material in its bulk form may no longer be biocompatible once transformed, processed and/or used in a neural implant. New materials and assembly techniques are not systematically discarded because of biosafety or biocompatibility standards, but their validation and approval may be long and laborious.

Considering electrode-tissue mechanical interactions, nearly all implantable electrodes, whether clinically approved or under development, are stiff with a mismatch in elastic moduli of several orders of magnitude compared with the targeted neural tissue (FIG. 2). Their insertion into living neural tissues triggers bidirectional interactions (that is, both implant-to-tissue and tissue-to-implant responses). Man-made implants induce insertion-related trauma to the host biological tissues, acute and then chronic inflammation, gliosis and disruption of the blood-brain barrier ${ }^{71}$. Conversely, the active and reactive biological medium affects the synthetic devices. These alterations include structural degradation ${ }^{5}$, material corrosion, insulating material failure $^{4}$ and electrode impedance fluctuation ${ }^{6}$ over periods of weeks to months. These changes compromise the stability and capability of recording or stimulating neural signals. Recent efforts in smart materials and soft bioelectronics aim at tackling the multiscale, multifunction mismatches between neural tissues and man-made implants. The majority of these approaches involve the design of flexible mechanical structures ${ }^{7-9}$, the engineering of elasticity in a stiff and rigid material ${ }^{7-9}$ and the integration of softer materials into implant ${ }^{20,72}$. Two main objectives are being jointly pursued with the 'soft neurotechnology' toolbox: minimizing the long-term FBR and implementing multiple recording and neuromodulation modalities in mechanically compliant structures.

\section{Reduced foreign body reaction}

The surgical insertion of a synthetic device in the vicinity of or into a neural tissue triggers an immediate FBR that continues throughout the in vivo lifetime of the neural implant. This acute inflammation will turn into a chronic neuroinflammatory response characterized by a complex interplay of molecular and cellular components. 
Persistent inflammatory stimuli exacerbate the chronic inflammation ${ }^{22}$.

Mechanical stiffness of conformal surface implants. The physical and material properties of the implant, as well as the site of implantation, affect the intensity and duration of inflammatory and healing processes. Static and rigid implants fail to accompany the natural microand/or macroscopic motion of the neural tissues. Several groups have now demonstrated that the mechanical differences between neural tissues and implant materials induce adverse strain fields in the immediate vicinity of the device, which may lead to irreversible tissue damage and electrode failure $e^{4,71,73,74}$.

Surface implants should conform as much as possible to the convoluted surface of the brain (FIG. 3), spinal cord or peripheral nerves (FIG. 4). Implant (macroscopic) compliance may be achieved through the use of ultrathin materials. The flexural stiffness, $D$, of a material is defined in equation 1 : the thinner the material, the more bendable it is ${ }^{75}$.

$$
D=\frac{E h^{3}}{12\left(1-v^{2}\right)}
$$

where $E$ is the elastic modulus, $h$ is the thickness of the material and $v$ is Poisson's ratio. For example, the use of ultrathin and flexible electrodes combined with micrometre-thick silicon transistor membranes has enabled recordings of electrocorticograms (EcoG) from inaccessible cortical areas such as the interhemispheric fissure $^{76,77}$.

To accommodate the dynamics of the neural tissue, the tensile stiffness of the implant is another crucial design parameter. The tensile stiffness determines the ability of the implant to reversibly expand and relax with the underlying tissue. Patterning extremely thin $(<10 \mu \mathrm{m})$ and stiff films into web-like systems improves the mechanical coupling between the implant and the underlying moving tissue. On mechanical loading, the mesh develops into 3D out-of-plane structures with engineered compliance. These 3D topologies have been exploited to fabricate stretchable metallic conductors ${ }^{78}$ that conform to the curved surface of the skin $^{79}$ and biological organs ${ }^{9,80}$. A transient packaging material (for example, silk) is usually added to coat the delicate open-mesh surface and to ease handling and surgical insertion ${ }^{76}$. However, the dimensions of the mesh ligaments should be carefully controlled. Indeed, even in a thin and narrow rectangular format, the stiff and non-elastic material may severely limit the motion of the underlying neural tissue, or debond and slide against it, which is likely to induce and exacerbate damage and neuroinflammation.

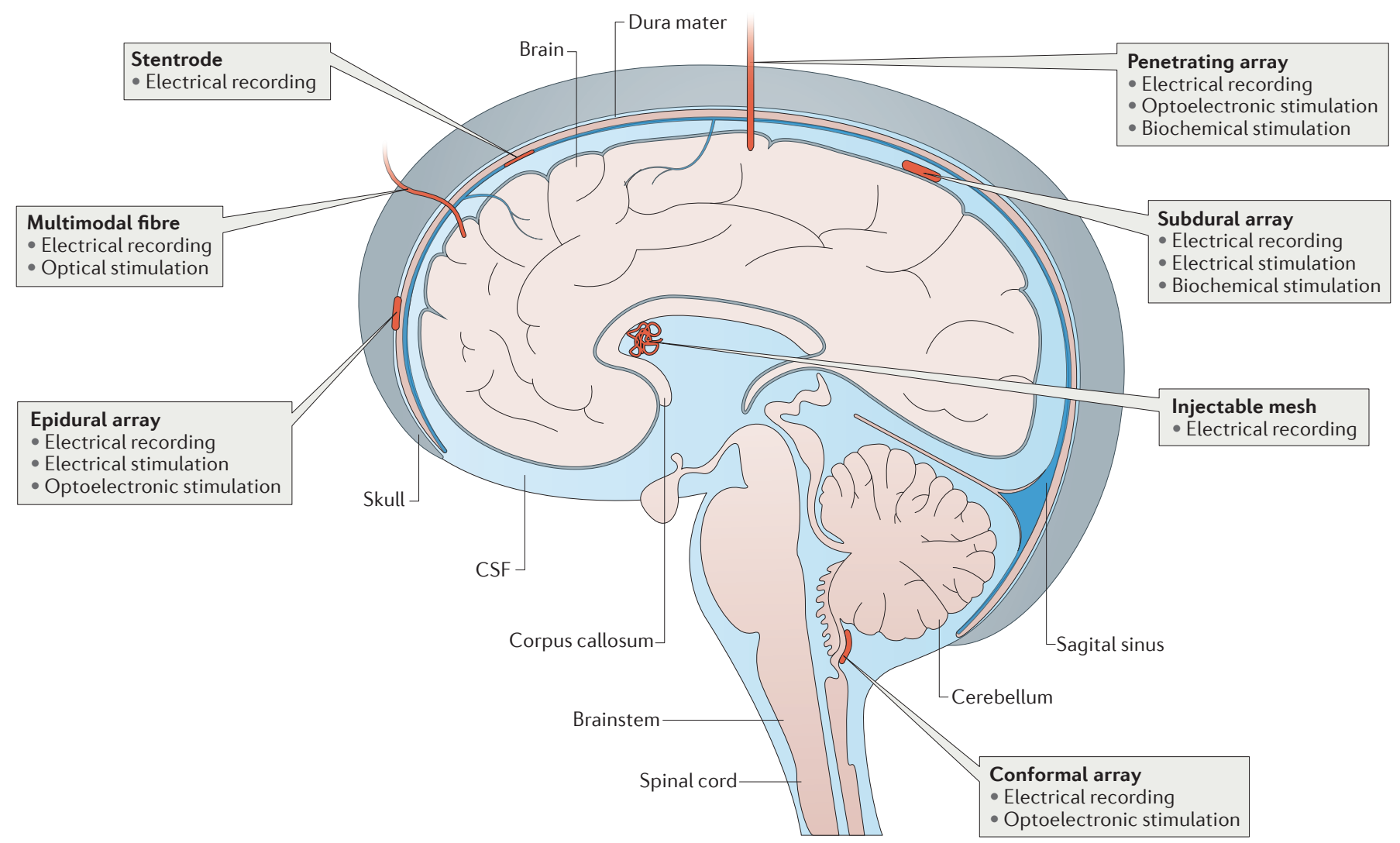

Figure 3 | Compliant and multimodal neural interfaces for the brain. Examples of implantable systems micro- and nanomachined to match the brain morphology and communicate efficiently using electrons, photons and chemical compounds. The recording and stimulation modalities are listed for each type of implant. References of the reported devices are: stentrode ${ }^{99}$; subdural arrays ${ }^{20}$, which include meshed $^{98}$ and bioresorbable arrays ${ }^{83}$; penetrating array ${ }^{117}$; multimodal fibre ${ }^{94,95,124}$; conformal array ${ }^{124}$; and injectable mesh ${ }^{97}$. 


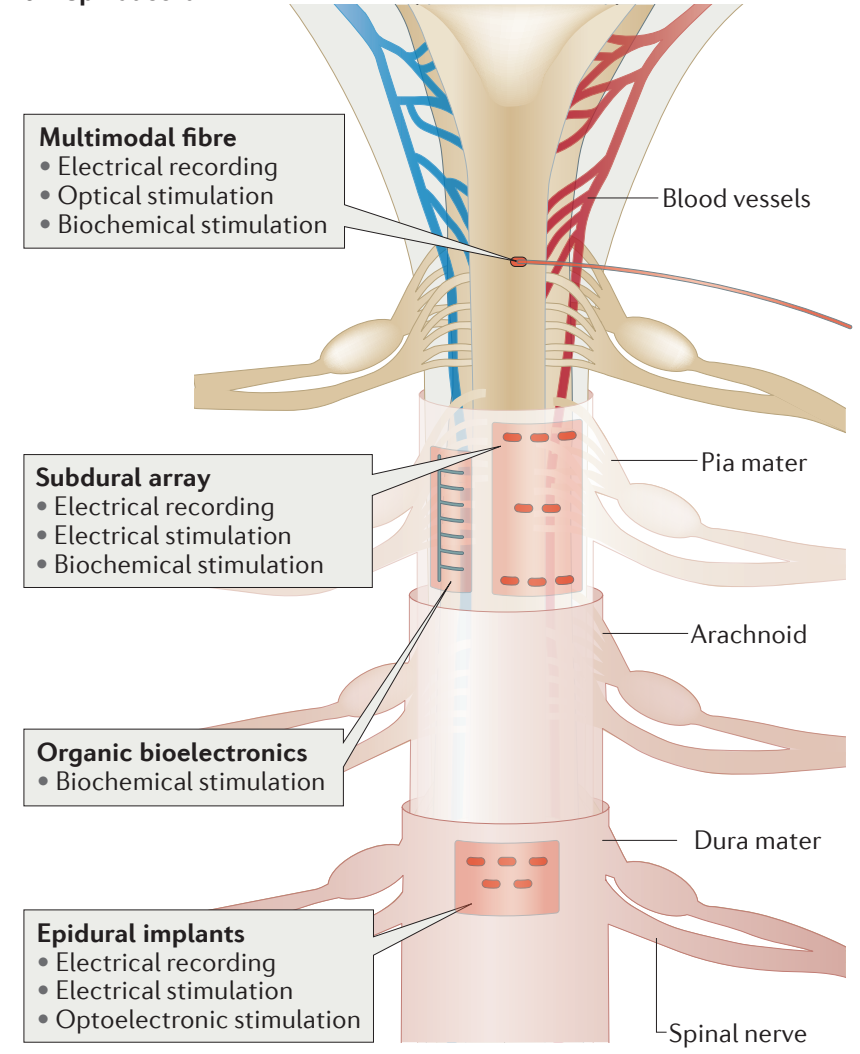

\section{b Peripheral nerve}

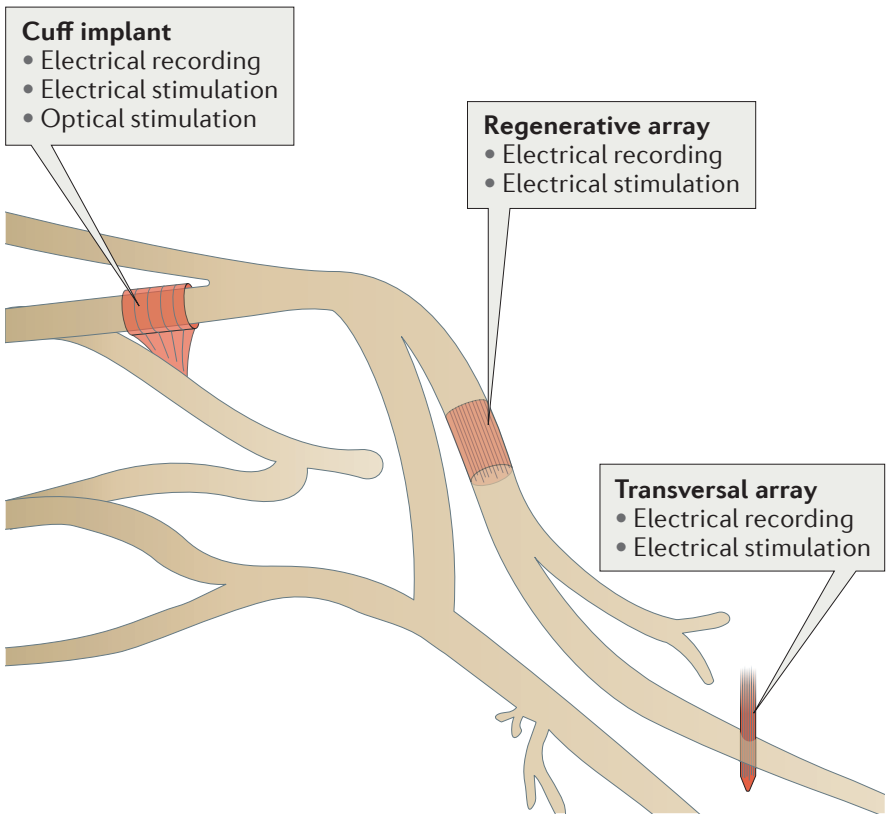

Figure 4 | Compliant and multimodal interfaces for the spinal cord and the peripheral nerves. Examples of implantable systems that have been micromachined to conform to the delicate surface of spinal tissues but minimally penetrate the tissues include multimodal fibres ${ }^{96}$, epidural arrays ${ }^{119}$, subdural arrays ${ }^{20}$ and organic bioelectronics ${ }^{113}$ (part a). Implants that mimic the macroscopic anatomy of peripheral nerves include cuff implants ${ }^{125,126}$, transversal arrays $^{127,128}$ and regenerative arrays ${ }^{129}$ (part b).

An alternative approach involves the manufacturing of the implant with elastic materials that render it soft and stretchable. When designed in a solid membrane format, this type of soft implant presents several advantages over thin films. First, the membrane is robust, which facilitates the handling of the implant and its surgical insertion into intricate regions of the CNS. For example, these membranes have been implanted chronically below the dura mater of the brain and spinal cord in rats. Second, these soft implants do not trigger the FBR. Indeed, a soft implant inserted subdurally prepared as a macroscopic membrane of elastomers with tensile mechanics matching that of dura mater ${ }^{20,81}$ proved nearly imperceptible to the underlying tissue even after weeks of implantation (FIG. 5a,b). This immediate and long-lasting biointegration is especially promising for ECoG arrays, which are typically used to monitor neural activity from the cortical surface.

Bioresorbable implants, prepared with ultrathin implantable electrode technology, also promise improved biointegration in acute and short-term transient neural interfaces. The nanometre-thick materials embedded in thin resorbable substrates yield devices with levels of mechanical flexibility necessary for conformal contact and stable interfaces with neural tissues for a preset duration $^{82,83}$.
Mechanical coupling of penetrating electrodes. The physical coupling between penetrating electrodes and neural tissues is even more complex given the trauma, local inflammation and astroglial scarring around the implant. Combinatorial strategies ranging from molecular biology to engineering are needed to design and implement long-term 'stealth' and functional implants. Here, we focus on the importance of the mechanical coupling between the implant and the tissue. A more exhaustive review on the various factors at work may be found in REF. 7 .

Macroscopically, the implant should be mechanically decoupled from (but electrically communicating with) its anchoring point, which is usually in the surrounding bone via a flexible electronic cable. This decoupling allows the probe to move with the neural tissue ${ }^{1,20}$. The mechanical response of the neural tissue to the insertion of a penetrating implant is influenced by the geometry of the probe and the speed at which the probe is inserted. Rapid insertion of sharp implants ${ }^{84}\left(>1 \mathrm{~mm} \mathrm{~s}^{-1}\right)$ made of stiff materials, such as silicon, is often favoured, because clean transection of fibres and cell membranes results in less distortion or tearing of neurons and vascular damage $^{85}$. A low insertion speed $\left(<200 \mu \mathrm{m} \mathrm{s}^{-1}\right)$ may, however, better account for the viscoelastic response of the brain and provide time for displaced capillaries and fibres to recover without rupture ${ }^{85}$. 

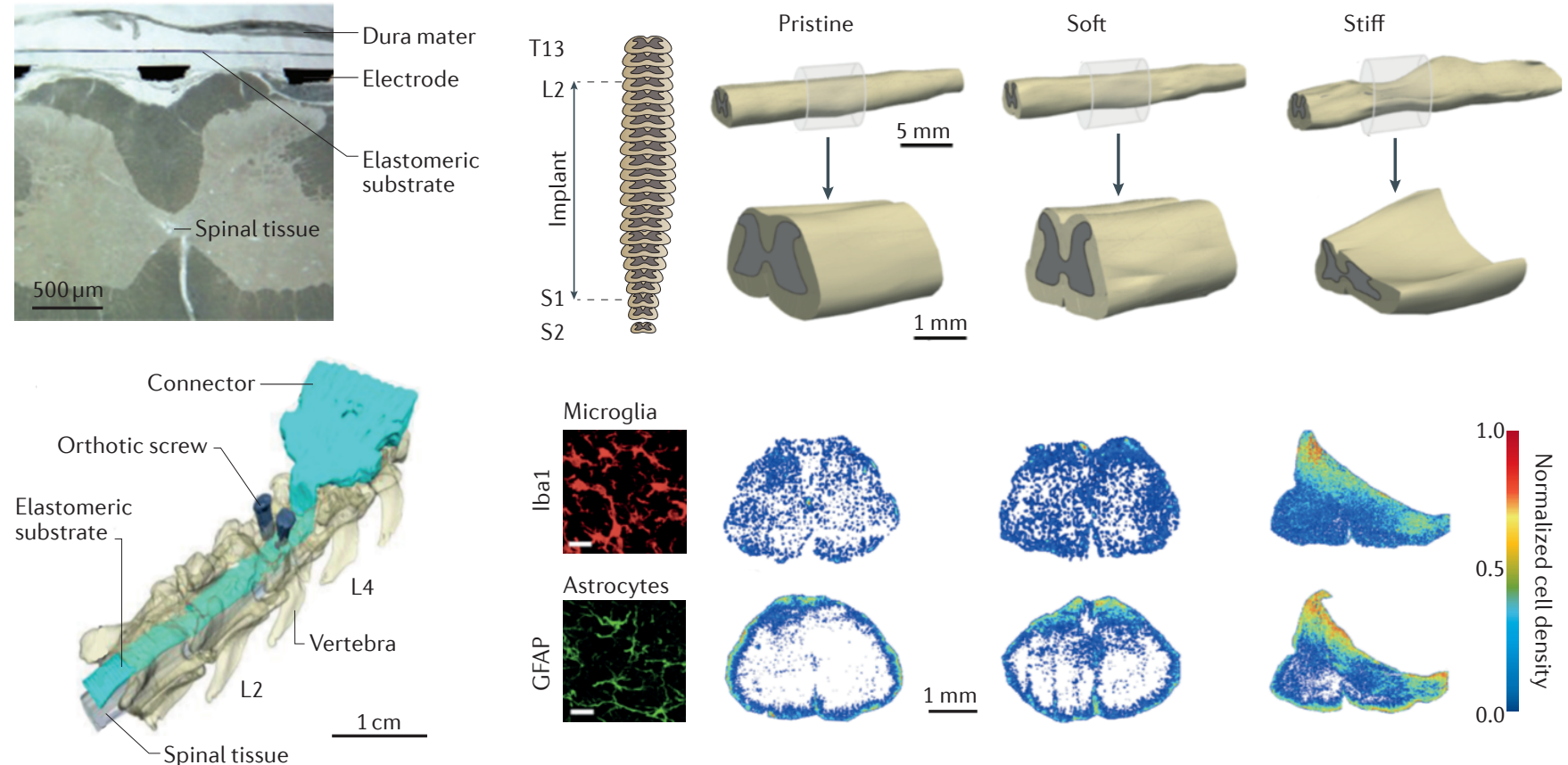

c Foreign body reactions around stiff and compliant shank implant
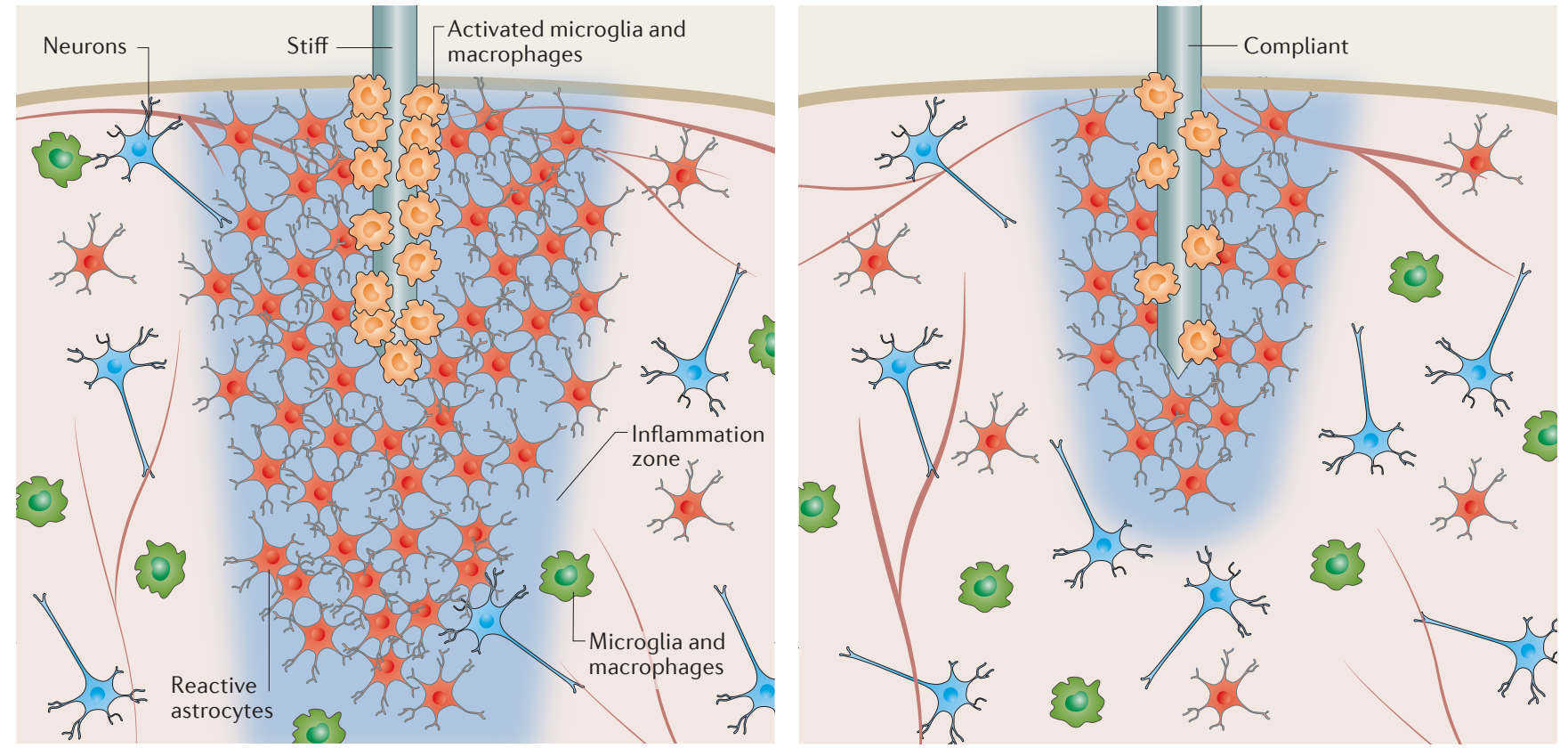

Reactive astrocytes (GFAP)

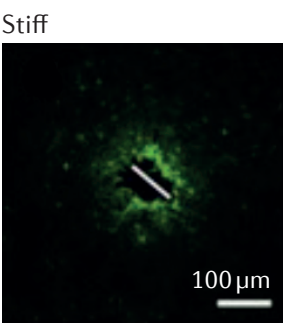

Microglia and macrophages

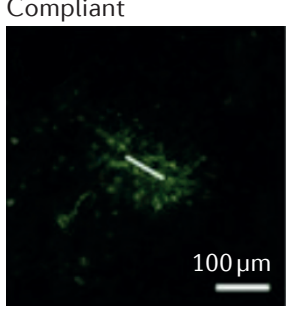

Stiff

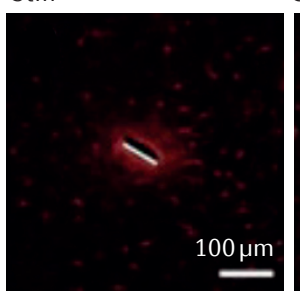

Compliant

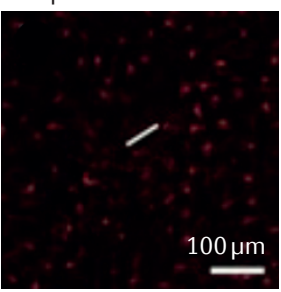

IgG immunoreactivity

Stiff

Compliant

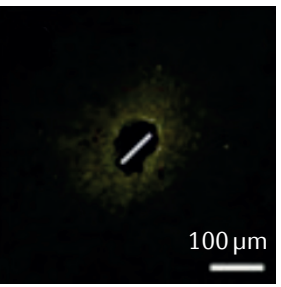

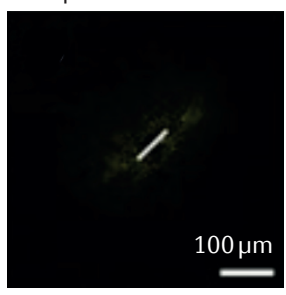


4 Figure 5 | Mechanosensitivity and foreign body reaction in the central nervous system. a | Biointegration of a subdural, soft implant at the surface of the spinal cord. The top panel depicts a cross-section of an electronic dura inserted in the spinal subdural space of a rat. The bottom panel is the corresponding reconstructed 3D micro-computed tomography scan ( 5 weeks after insertion) of the electronic dura covering the L2 to S1 spinal segments. b | Influence of soft and stiff implants on the spinal cord. The top panel illustrates 3D spinal cord reconstructions, including enhanced views 6 weeks after implantation. The photographs on the left of the bottom panel are of microglia (Iba1) and astrocyte (GFAP) staining, which reflect neuroinflammation (scale bars: $30 \mu \mathrm{m}$ ). The heat maps on the right show the normalized microglia (upper row) and astrocyte (lower row) density. c | The top two panels are schematic representations of the foreign body response around a stiff and a compliant penetrating shank into the cortex. Stiff implants (for example, poly(vinyl acetate)-coated silicon) induce increased gliosis, blood-brain barrier permeation and neurodegeneration in comparison to compliant materials (for example, poly(vinyl acetate)/tunicate cellulose nanocrystal nanocomposites) as shown in the photographs below. L2, L4, lumbar spinal discs; S1, S2, sacral spinal discs; T13, thoracic spinal disc; GFAP, glial fibrillary acidic protein; Iba1, ionized calcium-binding adaptor molecule 1 ; IgG, immunoglobulin G. Parts $\mathbf{a}$ and $\mathbf{b}$ are reproduced with permission from REF. 20, AAAS. Part $\mathbf{c}$ is adapted with permission from REF. 92, Institute of Physics. insulated with parylene $\mathrm{N}$ and conducting polymer poly(3,4-ethylenedioxythiophene) polystyrene sulfonate (PEDOT:PSS)-coated tips enable chronic recordings of single-unit activity in rats for several weeks ${ }^{94}$. Multi-electrode fibres prepared by thermal drawing a process borrowed from the fibre-optic industry - are a recent and promising design. In one study, microelectrodes ( $5 \mu \mathrm{m}$ in diameter) clustered in a single fibre (125 $\mu \mathrm{m}$ in diameter) were used to monitor neural activity at the single-unit level over a period of 2 months ${ }^{95}$. The fibres were prepared with insulating poly(phenylsulfone) or poly(etherimide) and conductive tin. In both examples, fibres remained intact in the brains of rats or mice for weeks of implantation, enabling clear recordings over this time range. Compared with multielectrode silicon shanks, the ultra-miniaturized fibres triggered less glial response, blood-brain barrier breach and tissue encapsulation in the vicinity of the probes ${ }^{95}$.

Injecting miniaturized and ultra-flexible electronics into brain tissue is another approach to interface the soft tissues in a minimally invasive procedure with reduced FBR (FIG. 3). A polyimide shank-like probe with very low bending stiffness and a small footprint can be temporarily mounted on a releasable carrier needle, which detaches once injected into the soft neural tissue ${ }^{87}$. Another design proposes a macroporous mesh made of narrow SU8 epoxy branches ${ }^{97}$. The high degree of porosity $(\sim 80 \%)$ of the implant together with subcellular feature sizes allow for a synthetic implant with extremely small bending stiffness and high malleability. The mesh format unfolds once in vivo into a 3D interface. Such a probe may be either injected through a hollow needle and then deployed in the liquid compartment of the brain (FIG. 3) (that is, the ventricles where the cerebrospinal fluid is produced ${ }^{97}$ or frozen in liquid nitrogen for rapid insertion into the cortex ${ }^{98}$. Cortical tissues carrying the macroporous ultra-flexible implants for several weeks display high biocompatibility as neural tissue invades the macroporous network. This response minimizes the electrode-neuron distance. Combined with a mature cardiovascular technology, the mesh design can also be used to form a stentrode. This novel implant is a combination of a stent, thin electrodes and transmitters injected into a vein via a catheter to travel in the brain through the bloodstream ${ }^{99}$.

\section{Engineering the local mechanical microenvironment.} Engineering of the mechanical (and surely biochemical) microenvironment at the surface of an implant may be needed to further modulate the FBR. It is increasingly appreciated that neurons and glial cells respond to the mechanical properties of their surroundings ${ }^{23,100}$ : neurons prefer softer regions over stiffer regions for growth and branching ${ }^{101}$; oligodendrocytes have an optimal stiffness of about $700 \mathrm{~Pa}$ (Young's modulus) for growth, proliferation, migration and differentiation ${ }^{35}$; astrocytes and microglia become activated on stiff surfaces ${ }^{102}$; and microglia migrate to regions that are stiffer (that is, with a Young's modulus $>10 \mathrm{kPa})^{103}$. One obvious approach to pacify cells in contact with an implant seems to be the use of materials with the same bulk modulus as CNS tissue. 
As a proof of concept, implantation of (non-electrically functional) soft hydrogel stripes (with an elastic modulus of $100 \mathrm{~Pa}$ ) caused less FBR than stripes of the same material that were two orders of magnitude stiffer ${ }^{102}$ (FIG. 5c). The slowly accumulating insight into the molecular mechanisms of the mechanosensing ${ }^{23,104-106}$ will allow drugable, pharmacological targets to be identified in order to better avoid FBR-causing mechanosensitivity of cells in contact with an implant. Slow release of suitable agonists could then be built into the implant surface to mechanically silence the cells in contact with it (that is, pharmacological stealthing of the implant). It is noteworthy that controlled in vivo release of bioactive agents, such as anti-inflammatory drugs ${ }^{107}$ and neurotrophic factors, can be integrated into conducting polymer materials ${ }^{108}$. However, the latter are probably most suitable for improving the electrode-neuron electrical interface ${ }^{109}$. The manufacturing of these materials allows integration with compliant carrier substrates and incorporation in gels.

Conducting polymers offer mixed electronic and ionic conductivity, promising improved charge transfer ${ }^{110}$. Several groups have reported on long-term neural interfaces based on conducting polymers, and extending the electrochemical stability of the polymers in vivo is an active topic of research ${ }^{94,11-113}$. PEDOT:PSS, which has a Young's modulus on the order of $100 \mathrm{MPa}$, is softer than standard electrode metals. Conducting polymer-hydrogel coatings hold significant promise as biointegrated interfaces, whereby the conducting polymer efficiently carries charges, and the hydrogel modulates the mechanical properties and may also enhance the drug-carrying capacity of the coatings ${ }^{109,114}$. In another interesting approach, tissue-engineered electrodes or 'live' electrodes, in which neuronal cells are encapsulated in a soft conducting hydrogel scaffold, are tailored to provide mechanical and biochemical properties that support cell growth and efficient electrochemical interfaces ${ }^{109}$.

\section{Multimodal soft neural implants}

Soft neural implants have, for the most part, been prepared with technologies initially developed for the microelectronic and telecommunication industries. In turn, the use of micro- and nanofabrication techniques in the manufacturing of neural implants in basic research has enabled the design of bidirectional interfaces, capable of distributed and local delivery of multiple inputs, such as electricity, light or drugs, and simultaneously monitoring the activity from one or several neurons. Currently, research implants for neuromodulation are primarily used to write into the nervous system using light to stimulate the neural tissues with improved specificity ${ }^{115,116}$, or a combination of two stimulation modalities (for example, electricity and pharmacology). Microelectrodes for monitoring neural activity are also often included in the multimodal stimulation interfaces. For example, injectable wireless optoelectronic implants embedded in ultrathin polyimide films incorporate independently addressable microscale inorganic light-emitting diodes that are multicoloured and co-located optical, thermal and
Figure 6 | Functions with compliant neural interfaces. a | A 64-channel, bioresorbable, actively multiplexed micro-electrocorticography array on the barrel cortex of a rat (left panel, visibly activated whiskers are marked in red) and estimated relative location of the recording array based on evoked potential results (right panel). $\mathbf{b} \mid$ Acute recordings of evoked potentials are made from the barrel cortex of a rat following stimulation of the whiskers (locations 1 and 2). The $8 \times 8$ electrode array captures the temporal characteristics (top panels) of the evoked potentials and their spatial distribution (bottom panels) on the cortex following two distinct stimulations. The colour map indicates the evoked potential size, interpolated across the array. c | Multifunctional fibres for simultaneous optical, electrical and chemical neuromodulation of the brain. The left panel is a photograph showing the macroscopic preform and the drawn microfibres containing microfluidic channels, electrodes and optical waveguides as the inset. The panels on the right show that the probes, implanted in the prefrontal cortex of Thy1-ChR2-YFP mice, enable co-localized optical stimulation (points in the spectra), injection of CNQX (synaptic inhibitor) and recordings of the corresponding electrophysiological activity at two months post-implantation. $\mathbf{d}$ | Rats were implanted with a spinal electronic dura mater covering the lumbosacral segments and received a severe spinal cord injury. The left panels are of the lesion reconstruction with photographs of the spinal cord injury shown below the schematic representation. In the right panels, bipedal locomotion under robotic support is recorded without and with electrochemical stimulation after three weeks of rehabilitation. Stick diagram decompositions of hindlimb movements are shown in the top panels. Leg muscle activity and hindlimb oscillations are shown below. CNQX, 6-cyano-7-nitroquinoxaline-2,3-dione; CPE, carbon-paste electrode. Parts $\mathbf{a}$ and $\mathbf{b}$ are from REF. 83, Nature Publishing Group. Part c is from REF. 95, Nature Publishing Group. Part $\mathbf{d}$ is reproduced with permission from REF. 20, AAAS.

electrophysiological sensors and actuators ${ }^{87}$ (FIG. 3). Complemented by a wireless powering platform, sophisticated and miniaturized implants have already communicated with the brain of freely moving mice, delivering light and pharmacology for several weeks ${ }^{117}$. These results demonstrate the good tolerance of the host medium and potential for long-term studies, which are essential for the development and understanding of neurotherapies. This technology has also recently been demonstrated in a bioresorbable format, enabling a range of acute and short-term applications for transient neural monitoring ${ }^{82,83}$ (FIG. 6a).

All-polymer fibre probes are another emerging approach for multimodal implants. These probes combine fibre-optical stimulation, drug delivery and electrical recording in a diameter of less than $300 \mu \mathrm{m}$ (REF. 95). Stable optical neuromodulation and the adjacent electrical recording of single-unit cortical neural activity have been demonstrated for up to 2 months post-implantation. In addition, controlled fluid infusion through the integrated hollow channels along the fibre allows for the delivery of not only drugs, but also viral vectors, including those used in optogenetics, and cell-selective 

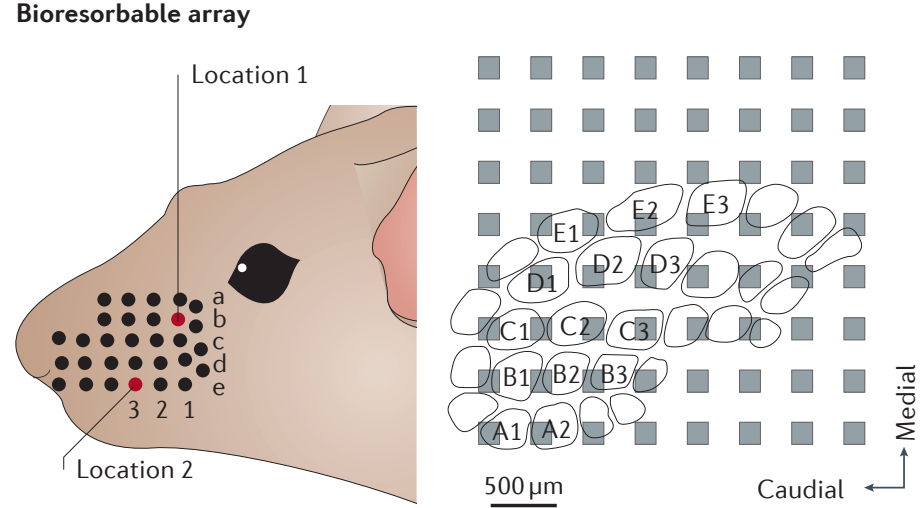

C Multimodal fibre

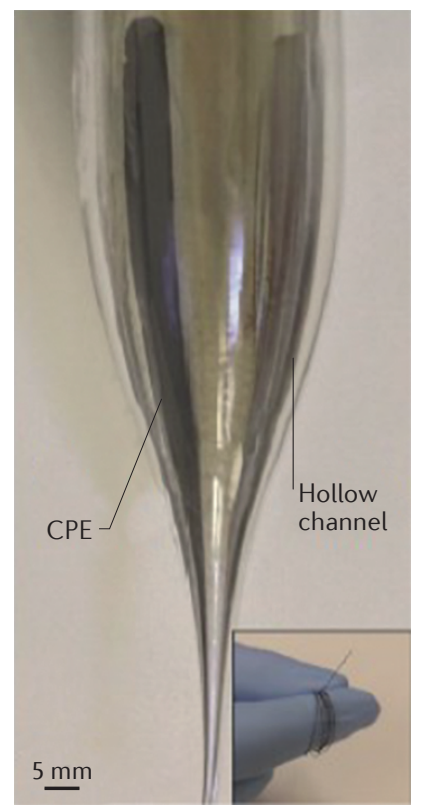

2 months

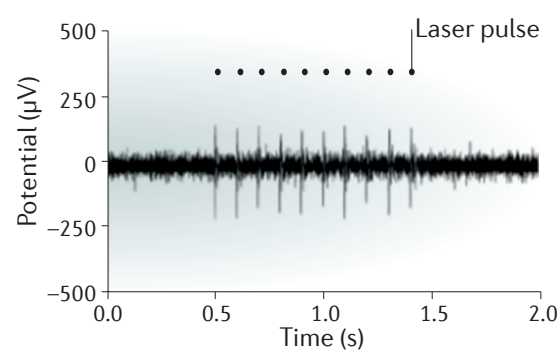

Before CNQX injection

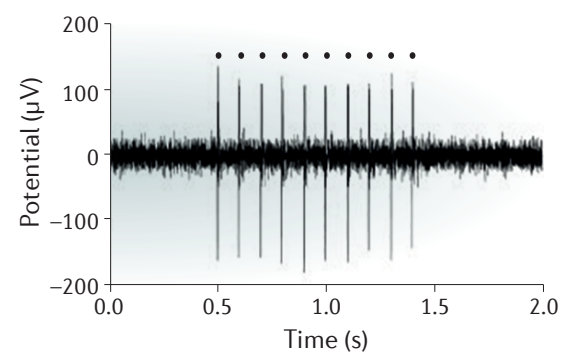

b
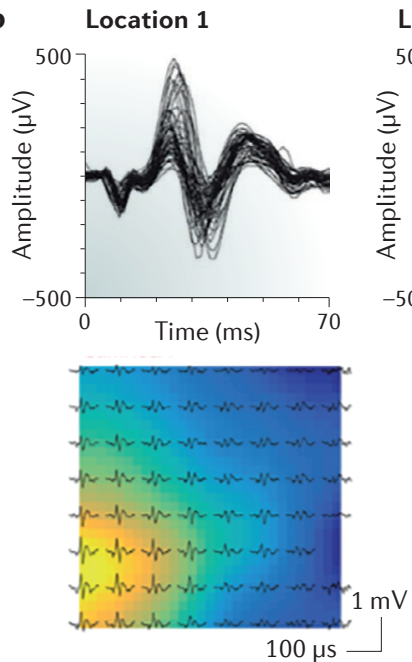

$100 \mu \mathrm{s}$
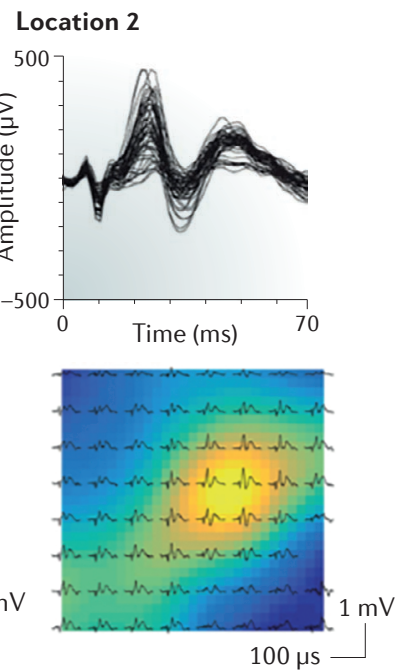

After CNQX injection

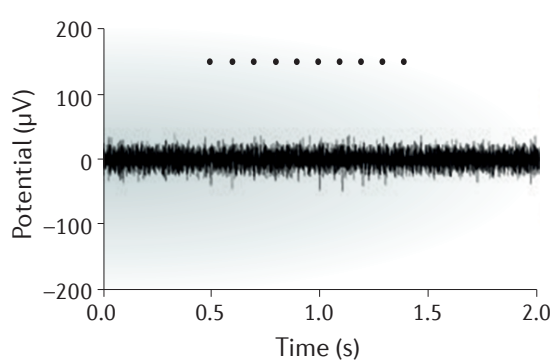

d Electrical stimulation with electronic dura mater
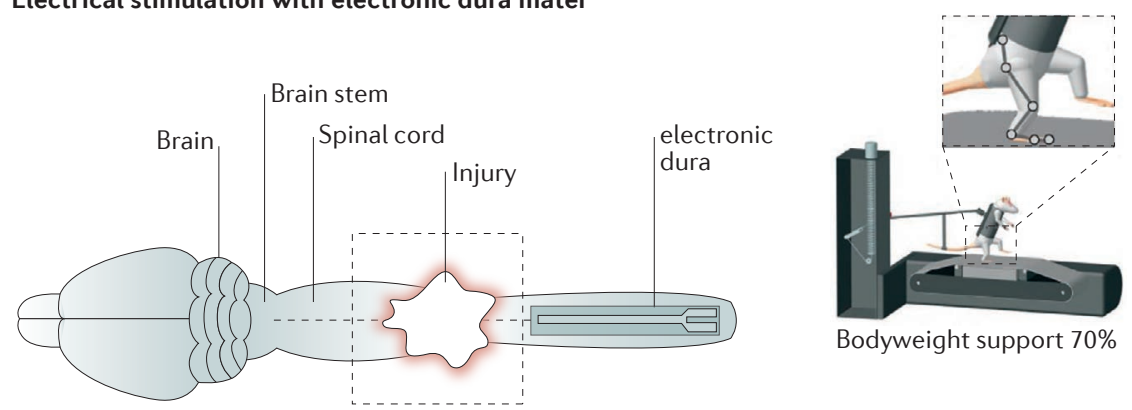

No stimulation

Electrochemical stimulation
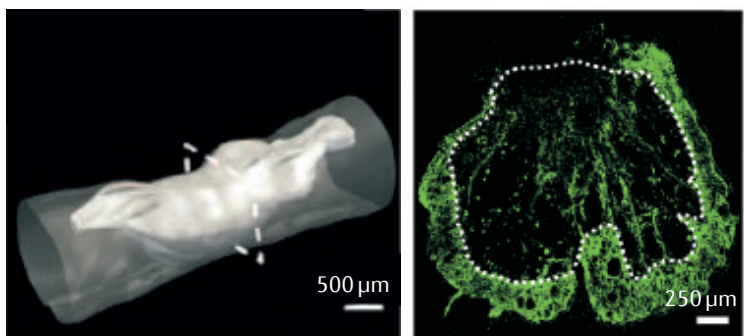

Flexor muscle activity

$$
\text { है। }
$$
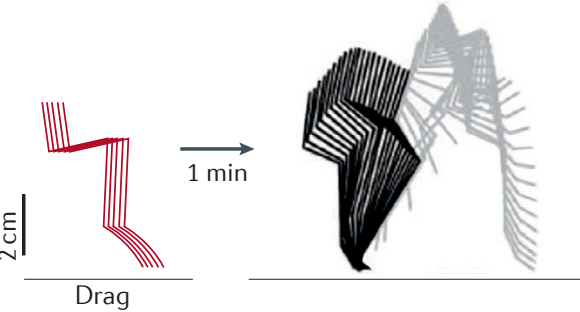

Extensor muscle activity

है।
ज़
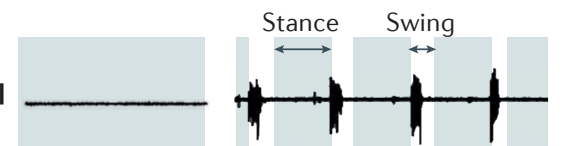

Limb kinematics
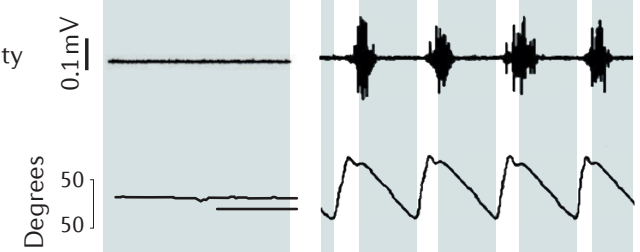
neurotoxins to neural tissues ${ }^{95}$. Consequently, ultraflexible polymer-based fibre probes appear as a promising biocompatible, multifunctional tool for dynamic optogenetic mapping of neural circuits in freely behaving animals (FIG. 6b).

Multimodal soft implants also accelerate progress in translational neuroprosthetic research. Electronic dura mater is a soft multifunctional implant prepared with elastomeric materials and designed to reside in the intrathecal space of the $\mathrm{CNS}^{20}$ (FIG. 6C). These implants have been used to deliver electrical stimulations over multiple locations of the spinal cord and to administer serotonergic agents that diffuse in the spinal tissue. The concurrent and co-localized electrical and chemical stimulations reactivate the spinal circuits below the injury ${ }^{118}$, which was shown to restore locomotion in paralysed rats. The embedded microfluidic channel provides highly localized delivery of minimally concentrated pharmacological agents. Electrical pulses delivered through the patterned microelectrode array to the underlying neural structures allow the control of the flexion and extension of the hindlimbs of the paralysed rats over the course of several weeks post-implantation ${ }^{119}$.

\section{A bright future for soft neurotechnology}

Manufacturing soft implants with brain-like materials is not yet possible; nevertheless, simple design rules (for example, based on thin, flexible materials, elastic membranes, miniaturization to cellular and even subcellular dimensions, multiscale $3 \mathrm{D}$ formats and colocalization of different types of transducers) now drive the research community to engineer and test soft neural implants with unprecedented biological integration and modalities.

Although the path to clinical translation remains challenging, the soft neurotechnologies reviewed in this article create possibilities for the development of new therapeutic treatments. The exciting results acquired in academic research, as well as the recent successes of companies such as Second Sight or Sapiens, which both engineered advanced implantable neuroprosthetic systems with a flexible neural interface at the heart of the system, have led to public funding (for example, the Brain Initiative in the USA) and unleashed private investment in start-ups developing implantable neuroprosthetic treatments.

Historically, neuroprosthetic treatments have been broadly divided into replacement and restoration strategies. Replacement primarily refers to the field of brain-machine interfaces, whereas restoration mainly focuses on neuromodulation therapies. The lack of biointegration in the neural tissue of today's implantable brain-machine interfaces impedes their vast dissemination into medical applications. As a consequence, neurointegrated implants, enabled in part by a more careful physical matching of the synthetic-biological interface and providing stable recordings of spiking activity for extended durations, would herald a new era for brainmachine interfaces, in which the prospect of restoring independent communication and assistive device control for people with paralysis would become reality. The stable delivery of electrical currents to the nervous system is considerably less challenging than the long-term recording of electrical signals from neural structures. It therefore comes as no surprise that neuromodulation therapies reached clinical fruition more rapidly than brain-machine interface technologies. For example, dopamine precursors and deep-brain stimulation of the basal ganglia circuitry have become common medical practices to alleviate cognitive and motor symptoms associated with Parkinson's disease.

Theoretically, the possibility of directly and precisely targeting specific brain ${ }^{120}$ and spinal cord ${ }^{119}$ structures with neuromodulation therapies can lead to remarkably more robust interventions. Soft electrode implants will have a pivotal role in enabling this approach. Indeed, chronic neuromodulation of targeted neural structures across and within the brain and spinal cord will require customized soft implants that match, or at least approach, neural tissue compliance, conform to the unique topography of these regions, and are capable of spatial and temporal selectivity. Moreover, the possibility to integrate microfluidic channels to deliver drugs locally creates exciting prospects of modulating neural excitability and boosting neuroplasticity. Local injections induce fewer side effects than systemic administration, which allows higher drug concentrations and thus more potent effects ${ }^{20}$. Many clinically approved compounds could be used in these applications.

The historical distinction between replacement and restoration strategies is becoming vague and ambiguous. The marriage of brain-machine interface technologies with neuromodulation therapies is occurring at a fast pace. Bidirectional implanted neuroprostheses combining recording and stimulating capabilities offer a unique opportunity to incorporate neural signals into closed-loop stimulation algorithms. The user then directly triggers and modulates the features of the therapy. For example, a quadriplegic person regained cortical control over isolated finger movements using a brain-controlled stimulation of hand muscles ${ }^{2}$. This approach $^{121}$ to neuroprosthetic designs is already supported in various research programs. For example, the aim of the ElectRx program is to develop intelligent pacemakers that personalize neuromodulation therapies through the closed-loop control of stimulation parameters. Next-generation implants with bidirectional and multimodal capabilities will have a central role in the success of these programs.

In summary, we are entering the era of personalized neuroprosthetics ${ }^{122}$. Because of the central role of the synthetic-biological interface, it is critical that materials scientists and engineers effectively design implant materials and structures that create an adequate biological and mechanical response with the soft neural tissue. We have gathered the first elements indicating that implant softness is essential to long-term use. It is an exciting time for materials scientists and engineers to discover how to further tailor implantable neuroprostheses and endow individuals suffering from traumatic or neurological disorders with personalized and life-changing therapeutic treatments. 
1. Hochberg, L. R. et al. Reach and grasp by people with tetraplegia using a neurally controlled robotic arm Nature 485, 372-375 (2012)

2. Bouton, C. E. et al. Restoring cortical control of functional movement in a human with quadriplegia. Nature 533, 247-250 (2016).

3. Barrese, J. C. et al. Failure mode analysis of silicon based intracortical microelectrode arrays in non-human primates. J. Neural Engineer. 10, 066014 (2013).

4. Barrese, J. C., Aceros, J. \& Donoghue, J. P. Scanning electron microscopy of chronically implanted intracortical microelectrode arrays in non-human primates. J. Neural Engineer. 13, 026003 (2016).

5. Sanchez, J. C., Alba, N., Batich, C. \& Carney, P. R. Structural modifications in chronic microwire electrodes for cortical neuroprosthetics: a case study. IEEE Trans. Neural Syst. Rehabil. Eng. 14, 217-212 (2006).

6. Sankar, V. et al. Electrode impedance analysis of chronic tungsten microwire neural implants: understanding abiotic vs. biotic contributions. Front Neuroeng. 7, http://dx.doi.org/10.3389/ fneng. 2014.00013 (2014).

7. Jorfi, M., Skousen, J. L., Weder, C. \& Capadona, J. R. Progress towards biocompatible intracortical microelectrodes for neural interfacing applications. J. Neural Eng. 12, 011001 (2015).

8. Jeong, J.-W. et al. Soft materials in neuroengineering for hard problems in neuroscience. Neuron $\mathbf{8 6}$ 175-186 (2015)

9. Lee, J. H., Kim, H., Kim, J. H. \& Lee, S.-H. Soft implantable microelectrodes for future medicine: prosthetics, neural signal recording and neuromodulation. Lab Chip 16, 959-976 (2016)

10. Prodanov, D. \& Delbeke, J. Mechanical and biological interactions of implants with the brain and their impact on implant design. Front. Neurosci. 10, http:// dx.doi.org/10.3389/fnins. 2016.00011 (2016).

11. Jones, E. G. \& Rakic, P. Radial columns in cortical architecture: it is the composition that counts. Cerebral Cortex 20, 2261-2264 (1984).

12. Bailey, S. A., Zidell, R. H. \& Perry, R. W. Relationships between organ weight and body/brain weight in the rat: what is the best analytical endpoint? Toxicol. Pathol. 32, 448-466 (2004).

13. Koo, B. B. et al. Age-related effects on cortical thickness patterns of the rhesus monkey brain Neurobiol. Aging 33, 200.e23-200.e31 (2012).

14. Herculano-Houzel, S. in In The Light of Evolution: Volume VI: Brain and Behavior Ch. 8 (eds Striedter, G F., Avise, J. C. \& Ayala, F. J.) 127-148 (National Academies Press, 2013)

15. Tallinen, T. et al. On the growth and form of cortical convolutions. Nat. Phys. 12, 588-593 (2016).

16. Wagshul, M. E., Eide, P. K. \& Madsen, J. R. The pulsating brain: a review of experimental and clinical studies of intracranial pulsatility. Fluids Barriers CNS 8, http://dx.doi.org/10.1186/2045-8118-8-5 (2011).

17. Harrison, D. E., Cailliet, R., Harrison, D. D., Troyanovich, S. J. \& Harrison, S. O. A review of biomechanics of the central nervous system - Part I: spinal canal deformations resulting from changes in posture. J. Manipulative Physiol. Ther. 22, 227-234 (1999).

18. Bashkatov, A. N. et al. Glucose and mannitol doffusion in human dura mater. Biophys. J. 85, 3310-3318 (2003).

19. Galasha, F. O. et al. A new type of recording chamber with an easy-toexchange microdrive array for chronic recordings in macaque monkeys. J. Neurophysiol. 105, 3092-3105 (2011)

20. Minev, I. R. et al. Biomaterials. Electronic dura mater for long-term multimodal neural interfaces. Science 347, 159-163 (2015).

21. Nicholson, K. J. \& Winkelstein, B. A. in Neural Tissue Biomechanics Ch. 10 (ed. Bilston, L. E.) 203-229 (Springer, 2011).

22. Polikov, V. S., Tresco, P. A. \& Reichert, W. M Response of brain tissue to chronically implanted neural electrodes. J. Neurosci. Methods 148, 1-18 (2005).

23. Franze, K., Janmey, P. A. \& Guck, J. Mechanics in neuronal development and repair. Annu. Rev. Biomed. Eng. 15, 227-251 (2013)

24. Ulrich, T. \& Kumar, S. in Mechanobiology Handbook 391-411 (CRC Press, 2011).

25. Bernick, K. B., Prevost, T. P., Suresh, S. \& Socrate, S. Biomechanics of single cortical neurons. Acta Biomater. 7, 1210-1219 (2011).

26. Lu, Y.-B. et al. Viscoelastic properties of individual glia cells and neurons in the CNS. Proc. Natl Acad. Sci. USA 103, 17759-17764 (2006).
27. Zou, S. et al. Force spectroscopy measurements show that cortical neurons exposed to excitotoxic agonists stiffen before showing evidence of bleb damage. PLOS One 8, e73499 (2013).

28. Grevesse, T., Dabiri, B. E., Parker, K. K. \& Gabriele, S Opposite rheological properties of neuronal microcompartments predict axonal vulnerability in brain injury. Sci. Rep. 5, 9475 (2015).

29. Bray, D. Mechanical tension produced by nerve cells in tissue culture. J. Cell Sci. 37, 391-410 (1979).

30. Dennerll, T. J., Joshi, H. C., Steel, V. L., Buxbaum, R. E. $£$ Heidemann, S. R. Tension and compression in the cytoskeleton of PC-12 neurites. II: Quantitative measurements. J. Cell Biol. 107, 665-674 (1988)

31. Dennerll, T. J., Lamoureux, P., Buxbaum, R. E. $\&$ Heidemann, S. R. The cytomechanics of axonal elongation and retraction. J. Cell Biol. 109, 3073-3083 (1989)

32. Bernal, R., Pullarkat, P. A. \& Melo, F. Mechanical properties of axons. Phys. Rev. Lett. 99, 018301 (2007)

33. MacDonald, R. B. et al. Müller glia provide essential tensile strength to the developing retina. J. Cell Biol. 210, 1075-1083 (2015)

34. O'Toole, M., Lamoureux, P. \& Miller, K. E. A physical model of axonal elongation: force, viscosity, and adhesions govern the mode of outgrowth. Biophys. $J$. 94, 2610-2620 (2008).

35. Jagielska, A. et al. Mechanical environment modulates biological properties of oligodendrocyte progenitor cells. Stem Cells Dev. 21, 2905-2914 (2012).

36. Lu, Y.-B. et al. Reactive glial cells: increased stiffness correlates with increased intermediate filament expression. FASEB J. 25, 624-631 (2011)

37. Vergara, D. et al. Biomechanical and proteomic analysis of INF- $\beta$-treated astrocytes. Nanotechnology 20, 455106 (2009)

38. Miller, W. J. et al. Mechanically induced reactive gliosis causes ATP-mediated alterations in astrocyte stiffness. J. Neurotrauma 26, 789-797 (2009).

39. Novak, U. \& Kaye, A. H. Extracellular matrix and the brain: components and function. J. Clin. Neurosci. 7 280-290 (2000)

40. Galtrey, C. M., Kwok, J. C. F., Carulli, D., Rhodes, K. E. \& Fawcett, J. W. Distribution and synthesis of extracellular matrix proteoglycans, hyaluronan, link proteins and tenascin-R in the rat spinal cord. Eur. J. Neurosci. 27, 1373-1390 (2008)

41. Barros, C. S., Franco, S. J. \& Müller, U. Extracellular matrix: functions in the nervous system. Cold Spring Harb. Perspect. Biol. 3, a005108 (2011).

42. Gaudet, A. D. \& Popovich, P. G. Extracellular matrix regulation of inflammation in the healthy and injured spinal cord. Exp. Neurol. 258, 24-34 (2014).

43. Swift, J et al. Nuclear lamin-A scales with tissue stiffness and enhances matrix-directed differentiation. Science 341, 1240104 (2013).

44. Syková, E. ¿ Nicholson, C. Diffusion in brain extracellular space. Physiol. Rev. 88, 1277-1340 (2008).

45. Hemphill, M. A., Dauth, S., Yu, C. J., Dabiri, B. E. \& Parker, K. K. Traumatic brain injury and the neuronal microenvironment: a potential role for neuropathological mechanotransduction. Neuron 85 1177-1192 (2015).

46. Pogoda, K. et al. Compression stiffening of brain and its effect on mechanosensing by glioma cells. New J. Phys. 16, 075002 (2014).

47. Javid, S., Rezaei, A. \& Karami, G. A micromechanical procedure for viscoelastic characterization of the axons and ECM of the brainstem. J. Mechan. Behav. Biomed. Mater. 30, 290-299 (2014).

48. Cheng, S., Clarke, E. C. \& Bilston, L. E. Rheological properties of the tissues of the central nervous system: a review. Med. Eng. Phys. 30, 1318-1337 (2008)

49. Goriely, A. et al. Mechanics of the brain: perspectives, challenges, and opportunities. Biomech. Model. Mechanobiol. 14, 931-965 (2015)

50. Fallenstein, G. T., Hulce, V. D. \& Melvin, J. W. Dynamic mechanical properties of human brain tissue. J. Biomech. 2, 217-226 (1969)

51. Ommaya, A. K. Mechanical properties of tissues of the nervous system. J. Biomech. 1, 137-138 (1968)

52. Chatelin, S., Constantinesco, A. \& Willinger, R. Fifty years of brain tissue mechanical testing: from in vitro to in vivo investigations. Biorheology 47, 255-276 (2010).

53. Koser, D. E., Moeendarbary, E., Hanne, J., Kuerten, S. \& Franze, K. CNS cell distribution and axon orientation determine local spinal cord mechanical properties. Biophys. J. 108, 2137-2147 (2015).
54. Franze, K. et al. Spatial mapping of the mechanical properties of the living retina using scanning force microscopy. Soft Matter 7, 3147-3154 (2011)

55. Budday, S. et al. Mechanical properties of gray and white matter brain tissue by indentation. J. Mechan. Behav. Biomed. Mater. 46, 318-330 (2015).

56. Elkin, B. S., Azeloglu, E. U., Costa, K. D. \& Morrison, B. Mechanical heterogeneity of the rat hippocampus measured by atomic force microscope indentation. J. Neurotrauma 24, 812-822 (2007).

57. Elkin, B. S. Ilankovan, A. I. \& Morrison, B. A detailed viscoelastic characterization of the $\mathrm{p} 17$ and adult rat brain. J. Neurotrauma 28, 2235-2244 (2011).

58. MacManus, D. B., Pierrat, B., Murphy, J. G. \& Gilchrist, M. D. Dynamic mechanical properties of murine brain tissue using micro-indentation J. Biomech. 48, 3213-3218 (2015).

59. Prange, M. T. \& Margulies, S. S. Regional, directional, and age-dependent properties of the brain undergoing large deformation. J. Biomech. Eng. 124, 244-252 (2002).

60. Christ, A. F. et al. Mechanical difference between white and gray matter in the rat cerebellum measured by scanning force microscopy. J. Biomech. 43 2986-2992 (2010).

61. Ichihara, K., Taguchi, T. \& Shimada, Y. Gray matter of the bovine cervical spinal cord is mechanically more rigid and fragile than the white matter. J. Neurotrauma 18, 361-367 (2001)

62. Shreiber, D. I., Hao, H. \& Elias, R. A. Probing the influence of myelin and glia on the tensile properties of the spinal cord. Biomech. Model. Mechanobiol. 8 , 311-321 (2009)

63. Schregel, K. K. et al. Demyelination reduces brain parenchymal stiffness quantified in vivo by magnetic resonance elastography. Proc. Natl Acad. Sci. USA 109, 6650-6655 (2012).

64. Elkin, B. S., Ilankovan, A. \& Morrison, B. Agedependent regional mechanical properties of the rat hippocampus and cortex. J. Biomech. Eng. 132, 011010 (2010)

65. Sack, l. et al. The impact of aging and gender on brain viscoelasticity. Neuroimage 46, 652-657 (2009).

66. de Rooij, R. \& Kuhl, E. Constitutive modeling of brain tissue: current perspectives. Appl. Mech. Rev. 68 010801-010823 (2016).

67. McKee, C. T., Last, J. A. \& Russell, P. Indentation versus tensile measurements of Young's modulus for soft biological tissues. Tissue Eng. Part B Rev. 17, 155-164 (2011)

68. Sridharan, A., Rajan, S. D. \& Muthuswamy, J. Longterm changes in the material properties of brain tissue at the implant-tissue interface. J. Neural Eng. 10, 066001 (2013).

69. Saxena, T., Gilbert, J., Stelzner, D. \& Hasenwinkel, J. Mechanical characterization of the injured spinal cord after lateral spinal hemisection injury in the rat. J. Neurotrauma 29, 1747-1757 (2012).

70. Estes, M. S. \& McElhaney, J. H. Response of brain tissue of compressive loading. Mech. Eng. 92, 58-61 (1970).

71. Goldstein, S. R. \& Salcman, M. Mechanical factors in the design of chronic recording intracortical microelectrodes. IEEE Trans. Biomed. Eng. 20, 260-269 (1973).

72. Chew, D. J. et al. A microchannel neuroprosthesis for bladder control after spinal cord injury in rat. Sci. Transl. Med. 5, 210 ra155 (2013).

73. Sharp, A. A., Ortega, A. M., Restrepo, D., CurranEverett, D. \& Gall, K. In vivo penetration mechanics and mechanical properties of mouse brain tissue at micrometer scales. IEEE Trans. Biomed. Eng. 56 45-53 (2009)

74. Hyunjung, L., Ravi, V. B., Wei, S. \& Marc, E. L. Biomechanical analysis of silicon microelectrodeinduced strain in the brain. J. Neural Engineer. 2 , 81-89 (2005)

75. Suo, Z., Ma, W. Y., Gleskova, H. \& Wagner, S. Mechanics of rollable and foldable film-on-foil electronics. Appl. Phys. Lett. 74, 1177-1179 (1999).

76. Kim, D.-H. et al. Dissolvable films of silk fibroin for ultrathin conformable biointegrated electronics. Nat. Mater. 9, 511-517 (2010).

77. Matsuo, T. et al. Intrasulcal electrocorticography in macaque monkeys with minimally invasive neurosurgical protocols. Front. Syst. Neurosci. 5, 34 (2011).

78. Guo, C. F., Sun, T., Liu, Q., Suo, Z. \& Ren, Z. Highly stretchable and transparent nanomesh electrodes made by grain boundary lithography. Nat. Commun. 5, 3121 (2014). 
79. Fan, J. A et al. Fractal design concepts for stretchable electronics. Nat. Commun. 5, 3266 (2014).

80. Xu, L. et al. 3D multifunctional integumentary membranes for spatiotemporal cardiac measurements and stimulation across the entire epicardium. Nat. Commun. 5, 3329 (2014).

81. Minev, I. R., Wenger, N., Courtine, G. \& Lacour, S. P. Research update: platinum-elastomer mesocomposite as neural electrode coating. APL Mater. 3, 014701 (2015).

82. Kang S.-K et al. Bioresorbable silicon electronic sensors for the brain. Nature 530, 71-76 (2016)

83. Yu, K. J., Kuzum, D., Hwang, S. W., Kim, B. H. \& Juul, $\mathrm{H}$. Bioresorbable silicon electronics for transient spatiotemporal mapping of electrical activity from the cerebral cortex. Nat. Mater. http://dx. doi. org/10.1038/nmat4624 (2016).

84. Edell, D. J., Toi, V. V., McNeil, V. M. \& Clark, L. D. Factors influencing the biocompatibility of insertable silicon microshafts in cerebral cortex. IEEE Trans. Biomed. Eng. 39, 635-643 (1992).

85. Bjornsson, C. S. et al. Effects of insertion conditions on tissue strain and vascular damage during neuroprosthetic device insertion. J. Neural Engineer. 3, 196-207 (2006).

86. Dryg I. D et al. Magnetically inserted neural electrodes: tissue response and functional lifetime. IEEE Trans. Neural Syst. Rehab. 23, 562-571 (2015).

87. Kim, T. et al. Injectable, cellular-scale optoelectronics with applications for wireless optogenetics. Science 12, 211-216 (2013)

88. Lee, K. et al. Polyimide based neural implants with stiffness improvement. Sens. Actuators B 102, 67-72 (2004).

89. Ware T et al Three-dimensional flexible electronics enabled by shape memory polymer substrates for responsive neural interfaces. Macromol. Mater. Eng. 297, 1193-1202 (2012)

90. Capadona, J. et al. A versatile approach for the processing of polymer nanocomposites with selfassembled nanofibre templates. Nat. Nanotechnol. 2 765-769 (2007).

91. Sridharan, A., Nguyen, J. K., Capadona, J. R. \& Muthuswamy, J. Compliant intracortical implants reduce strains and strain rates in brain tissue in vivo. J. Neural Eng. 12, 036002 (2015)

92. Nguyen, J. K. et al. Mechanically-compliant intracortical implants reduce the neuroinflammatory response. J. Neural Eng. 11, 056014 (2014).

93. Karnaushenko, D. et al. Biomimetic microelectronics for regenerative neuronal cuff implants. Adv. Mater. 27, 6797-6805 (2015).

94. Takashi, D. et al. Ultrasmall implantable composite microelectrodes with bioactive surfaces for chronic neural interfaces. Nat. Mater. 11, 1065-1073 (2012).

95. Canales, A. et al. Multifunctional fibers for simultaneous optical, electrical and chemical interrogation of neural circuits in vivo. Nat. Biotechnol. 33, 277-284 (2015)

96. Lu, C. et al. Polymer fiber probes enable optical control of spinal cord and muscle function in vivo. Adv Funct. Mater. 24, 6594-6600 (2014).

97. Liu, J. et al. Syringe-injectable electronics. Nat. Nanotechnol. 10, 629636 (2015).
98. Xie, C. et al. Three-dimensional macroporous nanoelectronic networks as minimally invasive brain probes. Nat. Mater. 14, 1286-1292 (2015).

99. Thomas, J. et al. Minimally invasive endovascular stent-electrode array for high-fidelity, chronic recordings of cortical neural activity. Nat. Biotechnol. 34, 320-327 (2016).

100. Discher, D. E., Janmey, P. \& Wang, Y. L. Cells feel and respond to the stiffness of their substrate. Science 310, 1139-1143 (2005)

101. Georges, P. C., Miller, W. J., Meaney, D. F Sawyer, E. S. \& Janmey, P. A. Matrices with compliance comparable to that of brain tissue select neuronal over glial growth in mixed cortical cultures. Biophys. J. 90, 3012-3018 (2006).

102. Moshayedi, P. et al. The relationship between glial cell mechanosensitivity and foreign body reactions in the central nervous system. Biomaterials 35, 3919-3925 (2014).

103. Bollmann, L. et al. Microglia mechanics: immune activation alters traction forces and durotaxis. Front. Cell. Neurosci. 9, 363 (2015).

104. Franze, K. \& Guck, J. The biophysics of neuronal growth. Rep. Progress Phys. 73, 094601 (2010).

105. Janmey, P. A. \& Miller, R. T. Mechanisms of mechanical signaling in development and disease. J. Cell Sci. 124, 9-18 (2011)

106. McWhorter, F. Y., Davis, C. T. \& Liu, W. F. Physical and mechanical regulation of macrophage phenotype and function. Cell. Mol. Life Sci. 72, 1303-1316 (2015).

107. Kozai, T. D. Y., Jaquins-Gerstl, A. S., Vazquez, A. L. Michael, A. C. \& Cui, X. T. Dexamethasone retrodialysis attenuates microglial response to implanted probes in vivo. Biomaterials 87, 157-169 (2016).

108. Chikar, J. A. et al. The use of a dual PEDOT and RGDfunctionalized alginate hydrogel coating to provide sustained drug delivery and improved cochlear implant function. Biomaterials 33, 1982-1990 (2012).

109. Aregueta-Robles, U. A., Woolley, A. J., Poole-Warren, L. A., Lovell, N. H. \& Green, R. A. Organic electrode coatings for next-generation neural interfaces. Front Neuroeng. 7, 15 (2014).

110. Khodagholy, D. et al. In vivo recordings of brain activity using organic transistors. Nat. Commun. 4, 1575 (2013)

111. Rivnay, J., Owens, R. s. n. M. \& Malliaras, G. G. The rise of organic bioelectronics. Chem. Mater. 26, 679-685 (2014).

112. Williamson, A. et al. Controlling epileptiform activity with organic electronic ion pumps. Adv. Mater. 27 3138-3144 (2015)

113. Jonsson, A. et al. Therapy using implanted organic bioelectronics. Sci. Adv. 1, e1500039 (2015)

114. Rylie, A. Green, Sungchul Baek, Poole-Warren, L. A. \& Martens, P. J. Conducting polymer-hydrogels for medical electrode applications. Sci. Tech. Adv. Mater. 11, 014107 (2010).

115. Montgomery, K. L., Iyer, S. M., Christensen, A. J., Deisseroth, K. \& Delp, S. L. Beyond the brain: optogenetic control in the spinal cord and peripheral nervous system. Sci. Transl. Med. 8, 337rv5 (2016).

116. Grosenick, L., Marshel, J. H. \& Deisseroth, K. Closed loop and activity-guided optogenetic control. Neuron 86, 106-139 (2015).
117. Jeong J J-W. et al Wireless optofluidic systems for programmable in vivo pharmacology and optogenetics. Cell 162, 1-13 (2015).

118. Courtine, G. et al. Transformation of nonfunctional spinal circuits into functional states after the loss of brain input. Nat. Neurosci. 12, 1333-1342 (2009).

119. Wenger, N. et al. Spatiotemporal neuromodulation therapies engaging muscle synergies improve motor control after spinal cord injury. Nat. Med. 22, 138-145 (2016)

120. McIntyre, C. C., Chaturvedi, A., Shamir, R. R. \& Lempka, S. F. Engineering the next generation of clinical deep brain stimulation technology. Brain Stimul. 8, 21-26 (2015).

121. Courtine, G. $\&$ Bloch, J. Defining ecological strategies in neuroprosthetics. Neuron 86, 29-33 (2015).

122. Borton, D., Micera, S., Millan, J. d. R. \& Courtine, G. Personalized neuroprosthetics. Sci. Transl. Med. 5, 210rv212 (2013).

123. Branner, A. \& Normann, R. A. A multielectrode array for intrafascicular recording and stimulation in sciatic nerve of cats. Brain Res. Bull. 51, 293-306 (2000).

124. Rubehn, B., Bosman, C., Oostenveld, R., Fries, P. \& Stiegltiz, T. S. A. MEMS-based flexible multichanne ECoG-electrode array. J. Neural Eng. 6, 036003 (2009).

125. Stieglitz, T., Beutel, H., Schuettler, M. \& Meyer, J. U. Micromachined, polyimide-based devices for flexible neural interfaces. Biomed. Microdevices 2, 283-294 (2000).

126. Towne, C., Montgomery, K. L., Iyer, S. M., Deisseroth, K. \& Delp, S. L. Optogenetic control of targeted peripheral axons in freely moving animals. PLoS One 8, e72691 (2013).

127. Boretius, T. et al. A transverse intrafascicular multichanne electrode (TIME) to interface with the peripheral nerve. Biosens. Bioelectron. 26, 62-69 (2010).

128. Raspopovic, S. et al. Restoring natural sensory feedback in real-time bidirectional hand prostheses. Sci. Transl. Med. 6, 222ra219 (2014).

129. Musick, K. M. et al. Chronic multichannel neural recordings from soft regenerative microchannel electrodes during gait. Sci. Rep. 5, 14363 (2015).

\section{Acknowledgements}

Financial support was provided by the Bertarelli Foundation (SPL), Starting Grants from the European Research Council (ERC 259419 ESKIN (SPL), ERC 261247, Walk Again (GC)), the European Community's Seventh Framework Program (CP-IP 258654, NeuWALK (GC)) and the Alexander-vonHumboldt Foundation (Alexander-von-Humboldt Professorship (JG)). The authors thank A. Goriely, K. Franze, P. Janmey, K Van Vliet, J. Fawcett, R. Franklin, M. Reimer and J. Bloch for useful discussions.

\section{Competing interests statement}

The authors declare no competing interests.

FURTHER INFORMATION

Tucker-Davis Technologies: http://www.tdt.com/corticalarrays.html 